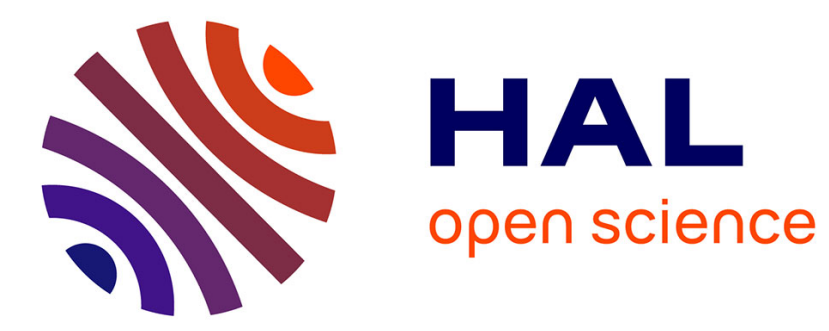

\title{
Le Protomagdalénien du Blot: étude typologique comparée
}

Bruno Bosselin

\section{To cite this version:}

Bruno Bosselin. Le Protomagdalénien du Blot: étude typologique comparée. Bulletin de la Société préhistorique française, 1992, 89 (3), pp.82-96. hal-00201596

\section{HAL Id: hal-00201596 \\ https://hal.science/hal-00201596}

Submitted on 14 Jan 2008

HAL is a multi-disciplinary open access archive for the deposit and dissemination of scientific research documents, whether they are published or not. The documents may come from teaching and research institutions in France or abroad, or from public or private research centers.
L'archive ouverte pluridisciplinaire HAL, est destinée au dépôt et à la diffusion de documents scientifiques de niveau recherche, publiés ou non, émanant des établissements d'enseignement et de recherche français ou étrangers, des laboratoires publics ou privés. 


\section{Le Protomagdalénien du Blot Étude typologique comparée}

par

Bruno BOSSELIN 


\title{
Le Protomagdalénien du Blot étude typologique comparée
}

\author{
par Bruno Bosselin
}

\section{LE SITE PRÉHISTORIQUE DU BLOT}

\section{I.1. Introduction}

Le site du Blot, à Cerzat (Haute-Loire), situé à égale distance des villages de Langeac et de Lavoute-Chilhac, est constitué d'une vaste falaise basaltique, haute d'une trentaine de mètres et longue de $300 \mathrm{~m}$ environ, orientée au Sud-Ouest. Elle domine un large méandre de l'Allier, entre les hameaux du Chambon de Cerzat et de Peyre, dans lequel sont conservées des terrasses formées d'alluvions anciennes. Le gisement préhistorique s'étend le long de la partie Est de cette coulée de basalte à une centaine de mètres de la rive droite de la rivière.

\section{I.2. Historique}

Le site archéologique du Blot a été découvert lors de l'hiver 1934-1935 par M. Estival, agriculteur au Chambon, qui avait entrepris une profonde tranchée aquifère perpendiculaire au fond de l'abri. Il recueillit, dans ce qui devait constituer le chantier III des fouilles de H. Delporte, une série lithique et osseuse abondante qu'il confia, plus tard, au professeur Mayet à l'Institut de Géologie de la Faculté de Lyon.

J. Combier effectua, en 1956-1957, un premier sondage préliminaire qui confirma l'intérêt archéologique du gisement. Il détermina une stratigraphie et distingua plusieurs ensembles : un Magdalénien terminal, une industrie archaïque sur quartz et surtout de nombreux niveaux d'habitat caractérisés par un riche outillage comprenant de belles lames retouchées d'aspect aurignacien mais dont la faiblesse numérique empêchait toute attribution culturelle.

Près de 10 ans plus tard, H. Delporte reprit l'étude de l'ensemble du site. La fouille du chantier I, étendue sur $25 \mathrm{~m}^{2}$, permit, durant trois années, la mise au jour d'une industrie lithique datée d'un Magdalénien terminal auvergnat riche en microlithes (Delporte, 1966). Ouvert à environ 30 mètres au Sud-Ouest, le chantier II ne livra que de très rares pièces atypiques. Situé à l'extrémité Sud-Est du site (sur l'emplacement même du premier sondage de J. Combier et de M. Estival), le chantier III occupera rapidement une superficie de près de $60 \mathrm{~m}^{2}$. Dans la partie supérieure, les recherches se révélèrent décevantes. La série recueillie, quoique moins riche, se rapportait à un Magdalénien final, semblable au précédent. Par contre, les couches profondes témoignèrent d'un intérêt exceptionnel puisque renfermant des niveaux stratifiés du Badegoulien, du Protomagdalénien et du Périgordien (Delporte, 1969).

\section{I.3. Stratigraphies du chantier III}

Dans une étude préliminaire, H. Delporte souligne l'extrême complexité de la stratigraphie du chantier III. En effet, les sédiments qui constituent le remplissage ne sont pas, comme en Périgord, très nettement différenciés. Ainsi, au fur et à mesure de l'avancement des travaux, les fouilleurs ont été amenés à modifier légèrement la numérotation des couches. Par ailleurs, parallèlement à celles-ci, dans certaines zones périphériques de la fouille, une dénomination annexe a été fournie, permettant un meilleur raccordement dans un site difficile à appréhender (tabl. 1). Nous ne présenterons pas ici en 


\begin{tabular}{|c|c|c|c|c|c|c|c|c|c|}
\hline \multicolumn{3}{|c|}{ STRATIGRAPHIES } & \multicolumn{6}{|c|}{ BANDES LATERALES } & \multirow[b]{2}{*}{ PROFILS } \\
\hline 1969 & 1970 & 1971 & $\mathrm{~A} / \mathrm{F}$ & G & $\mathrm{H}$ & $\mathrm{J}$ & $\mathrm{K} / \mathrm{L}$ & $\mathrm{N}$ & \\
\hline $\begin{array}{l}17 \\
17 \mathrm{~b} \\
21\end{array}$ & & 21 & & & & & & & \\
\hline $\begin{array}{l}22 \text { foy. } \\
22 \text { sup. } \\
22 \text { bases } \\
23 \text { fory. } \\
23\end{array}$ & & $\begin{array}{c}23 \text { foy. } \\
23 \\
24-25 \\
25 \\
26(28)\end{array}$ & & & & & & & ENSEMBLE 1 \\
\hline $\begin{array}{l}23 \text { a sup. } \\
23 a \text { corps }\end{array}$ & $\begin{array}{l}24-25 \\
26\end{array}$ & $\begin{array}{l}27 \\
27 \mathrm{c} \\
28\end{array}$ & (A5) & GA & & & & $\mathrm{NA}$ & ENSEMBLE 2 \\
\hline $\begin{array}{c}23 a \text { base } \\
23 \mathrm{a} / \mathrm{b}\end{array}$ & & $\begin{array}{l}30 \\
31\end{array}$ & $\begin{array}{l}\mathrm{A} 6 \\
\mathrm{~A} 6\end{array}$ & $\begin{array}{c}G A \\
G A\end{array}$ & & JA & кв & & ENSEMBLE 3 \\
\hline $\begin{array}{c}23 \mathrm{~b} \\
23 \mathrm{c} \\
\\
\begin{array}{l}23 \mathrm{dc} \\
23 \mathrm{~d} d\end{array}\end{array}$ & $\begin{array}{c}27-27 c \\
28 \\
29 \\
29 c \\
29 / 30 \\
30-30 c \\
31\end{array}$ & $\begin{array}{c}32-32 \mathrm{c} \\
33 \\
\\
33 \\
34 \mathrm{c}\end{array}$ & $\begin{array}{l}\text { A6 } \\
\text { (A7) } \\
\text { (AB) }\end{array}$ & $\begin{array}{c}\mathrm{GA}-\mathrm{GB} \\
\mathrm{GB} \\
\mathrm{GB} \\
\mathrm{GB}\end{array}$ & $\begin{array}{l}\mathrm{HA} \\
\mathrm{HB} \\
\mathrm{HC} \\
\mathrm{HD} \\
\mathrm{HD}\end{array}$ & 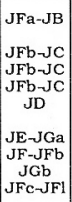 & \begin{tabular}{|c} 
Кв \\
KB-LH \\
LH \\
LH
\end{tabular} & $\begin{array}{l}\mathrm{NB} \\
\text { (NC) } \\
\text { ND } \\
\text { ND } \\
\text { ND }\end{array}$ & ENSEMBLE 4 \\
\hline $\begin{array}{c}23 \mathrm{~d}-23 \mathrm{e} \\
24 \\
25 \\
\\
26\end{array}$ & 32 & $\begin{array}{l}35 \\
36\end{array}$ & $\begin{array}{l}(\mathrm{Ag}) \\
\mathrm{Fe} \\
(\mathrm{FF})\end{array}$ & $\begin{array}{c}\text { GE-GB } \\
\text { GF-GH }\end{array}$ & $\begin{array}{l}\mathrm{HE} \\
\mathrm{HE} \\
\mathrm{HE} \\
\mathrm{HF}\end{array}$ & \begin{tabular}{|c|}
$\mathrm{JGc}$ \\
$\mathrm{JGG} J \mathrm{JGd}$ \\
$\mathrm{JH}-\mathrm{JI}$ \\
$\mathrm{JI}$ \\
$\mathrm{JI}$
\end{tabular} & & & \\
\hline $\begin{array}{c}27 \\
28 \\
29 \\
\\
29 \mathrm{c} \\
30 \text { foy. } \\
30\end{array}$ & $\begin{array}{l}34 \\
35\end{array}$ & $\begin{array}{c}37-37 b \\
37 c-37 d \mathrm{~d} \\
39 \\
39 \mathrm{db} \\
\\
39 \mathrm{c} \\
39 \mathrm{~d} \\
40 \\
40 \mathrm{C}\end{array}$ & $\begin{array}{l}\mathrm{Fg} \\
\mathrm{Fg}\end{array}$ & $\begin{array}{c}\mathrm{GH} \\
\mathrm{GI} \\
\mathrm{GJ}\left({ }^{*}\right) \\
\\
\mathrm{GKc} \\
\mathrm{GL} \\
\mathrm{GL} \mathrm{GM} \\
\mathrm{GMC}\end{array}$ & $\begin{array}{c}\text { HF base } \\
\text { HK } \\
\\
\mathrm{HK} \\
\mathrm{HK} \\
\mathrm{HM} \\
\mathrm{HM} \\
\mathrm{HMC}\end{array}$ & \begin{tabular}{|c|}
$\mathrm{JJ}$ \\
$\mathrm{JK}$ \\
$\mathrm{JKK}$ \\
$\mathrm{JKC}$ \\
$\mathrm{JL}$ \\
$\mathrm{JMC}(*)$ \\
$\mathrm{JN}$ \\
$\mathrm{JNc}$
\end{tabular} & & $\begin{array}{l}\mathrm{NF} \\
\mathrm{NH}\end{array}$ & PERIGORDIEN \\
\hline
\end{tabular}

Tableau 1 - Stratigraphies comparées du site du Blot.

détail les principales indications publiées d'une part par H. Delporte en 1969 (Delporte, 1969) et, d'autre part, plus récemment, par J. Virmont dans sa thèse (Virmont, 1981), complétées par les remarques géologiques de F. Moser puis D. Marguerie (Moser, 1976 ; Marguerie, 1982).

Toutefois, les auteurs s'accordent pour reconnaître l'existence de quatre grands ensembles culturels :

- Dans le haut de la stratigraphie, de la couche 3 à 7 , des niveaux riches datés d'un Magdalénien terminal.

- Les niveaux 8 à 20 livrent une industrie archaïque sur quartz et silex attribuable au Badegoulien.

- Une série de sols d'occupation (couches 23 à 34) du Protomagdalénien.

- Un ensemble inférieur, riche en lamelles à dos et chutes de burin : il s'agit d'un Périgordien supérieur.

\section{I.4. Corrélations entre les différentes stratigraphies}

L'étude typologique et statistique du matériel ne pouvant s'appuyer sur les multiples appellations précédentes, nous avons été amenés à utiliser la technique des profils pour appréhender la stratigraphie du Protomagdalénien. En d'autres termes, nous avons découpé la surface de fouille en transepts de $25 \mathrm{~cm}$ de largeur, sensiblement dans l'axe de la paroi. Chaque objet sera ensuite projeté sur un plan vertical, lui-même parallèle à la falaise, fournissant ainsi une coupe locale de la bande de terrain considérée. Cette méthode possède un triple avantage : permettre un suivi fin et précis de la succession des niveaux, reconnaître les zones d'occupation maximale et enfin, déterminer les pendages des couches (aussi bien sur les coupes sagittales que frontales).

En conséquence, nous avons reconnu quatre niveaux dans le Protomagdalénien du Blot:

- Ensemble 4 : Première occupation protomagdalénienne, correspondant aux couches $31,32,33$ et 34.

- Ensemble 3 : Seconde occupation protomagdalénienne, regroupant principalement la couche 30.

- Ensemble 2 : Troisième occupation protomagdalénienne ayant livré des structures d'habitat très nettes. Il correspond en majorité à la couche 27 et regroupe partiellement les couches 28 et 29.

- Ensemble 1 : Dernière occupation protomagdalénienne, plus sporadique et moins homogène, montrant une densité maximale des vestiges à la périphérie de la fouille.

\section{LE PROTOMAGDALENIEN DU BLOT}

\section{II.1. Les caractéristiques typologiques du Proto- magdalénien du Blot}

L'industrie lithique du Protomagdalénien du Blot a été étudiée suivant la liste-type de D. de Sonneville-Bordes et J. Perrot (Sonneville-Bordes et Perrot, 1954, 1955, 1956). Les décomptes effectués pour les quatre niveaux d'occupation sont fournis dans le tableau 2. Dans l'ensemble, les groupes typologiques principaux $(\mathrm{G}-\mathrm{B}$ - Bd - Bt - Pointe de la Gravette - Lame retouchée - Pièce esquillée Outils « archaïque »- Microlithe) montrent des variations relatives extrêmement réduites. Le site du Blot à Cerzat, dont l'homogénéité peut être conjointement attribuée à la qualité de l'échantillonnage issu des fouilles et à une évolution interne assez lente, présente donc les caractéristiques suivantes : 


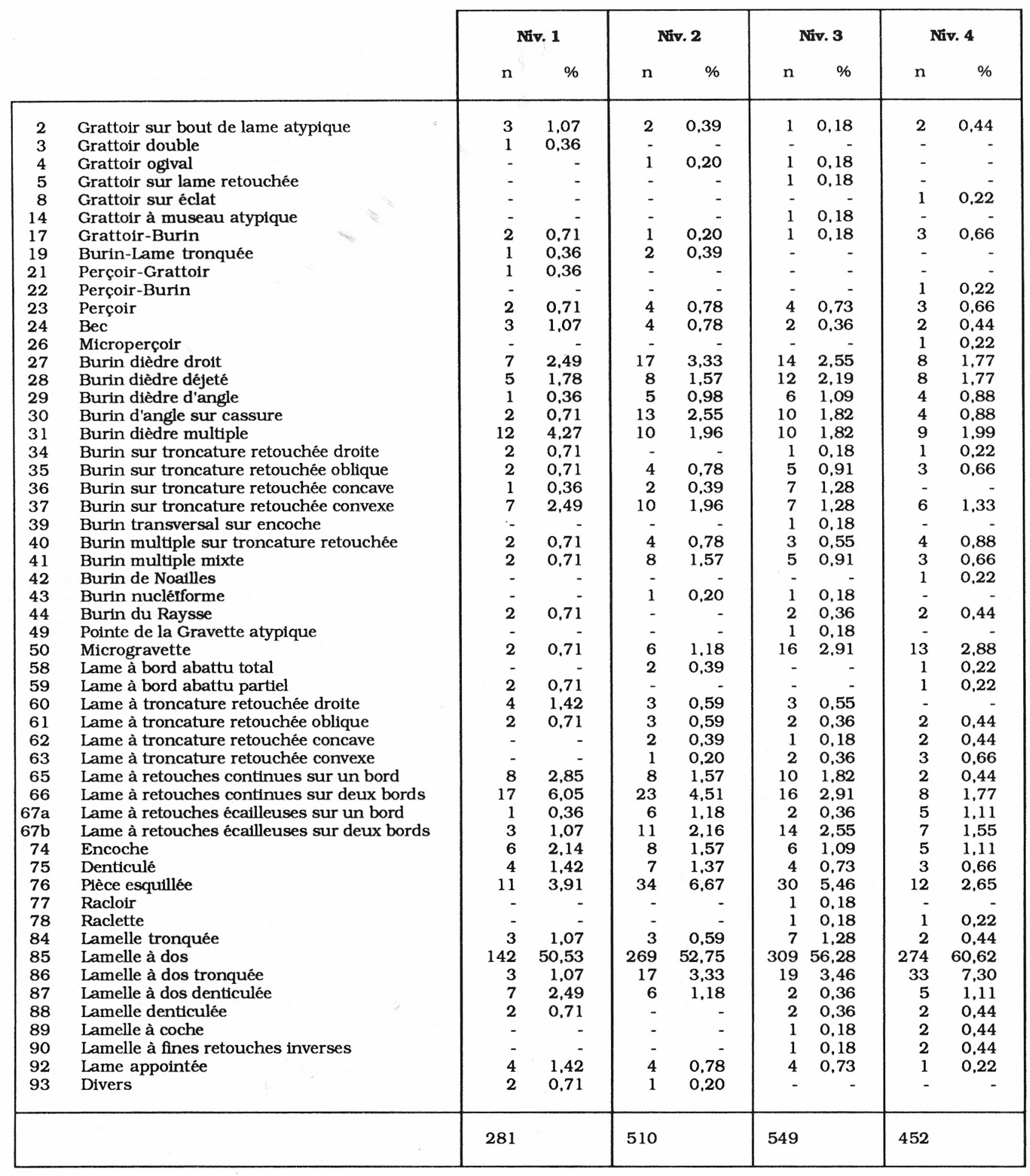

Tableau 2 - Décomptes typologiques de l'industrie lithique du Protomagdalénien du Blot.

- Extrême rareté des grattoirs $(0,55$ à 1,42\%), surtout sur extrémité de lame non retouchée, à front plat irrégulier et délimité par un front de mau- vaise facture. Notons également le très faible développement des autres types (aurignacien, double, sur éclat ou ogival). 
- Les outils composites sont rares, et regroupent principalement des grattoirs-burins, des burinslames tronquées, des perçoirs-grattoirs et des perçoirs-burins.

- Les perçoirs et becs, en quantité équivalente, associent fréquemment une troncature oblique et une encoche opposée. Notons également la présence d'un seul microperçoir sur lamelle.

- Fréquence moyenne des burins $(11,50$ à $16,09 \%)$, parmi lesquels les dièdres $(7,30$ à $10,39 \%$ ) dominent largement les types sur troncature retouchée $(3,10$ à $4,98 \%)$. Les burins dièdres d'axe (médians ou déjetés) l'emportent, parfois nettement, sur les burins d'angle (dièdres d'angle ou d'angle sur cassure). Les fractures de Corbiac, toujours présentes, attestent d'un refaçonnage du biseau. Les burins dièdres multiples sont très nombreux (de 19 à $44 \%$ des $\mathrm{Bd}$ ), surtout pour la couche 1. Les burins sur retouche, plus rares, présentent une variabilité plus faible : les troncatures convexes ou obliques étant les plus fréquentes. Il existe un seul burin transversal sur encoche. Les burins sur troncature retouchée multiples apparaissent moins développés (13 à $28 \%$ des Bt), ainsi que les burins multiples mixtes. Cet outil est, à la fin du cycle gravettien, un marqueur typologique, culturel et chronologique très précis : burin de Noailles, burin du Raysse et burin de Corbiac par exemple. Le site du Blot ne déroge pas à la règle puisque nous observons sporadiquement la présence de ces différents types. Les burins du Raysse se rencontrent dans trois niveaux tandis que le seul burin de Noailles a été recueilli dans l'ensemble le plus ancien (niveau 4). Leur apparition, vraisemblablement aléatoire, ne permet pas de conforter le modèle d'une substitution progressive des burins de Noailles par les burins du Raysse (Rigaud, 1982 ; Movius et David, 1970). L'échantillon recueilli (7 pièces sur 3 couches) n'autorise guère qu'un traitement de présence/absence, bien moins précis et riche en informations qu'un tableau d'effectifs ou de contingence (Djindjian, 1980 ; Bosselin et Djindjian, 1988 [1990]). Il faut signaler enfin l'importance de la retouche tertiaire du biseau, surtout sur les burins dièdres.

- Les pointes et micropointes de la Gravette, si elles ne présentent pas le développement observé dans les couches périgordiennes sous-jacentes montrent des fréquences non négligeables (de 0,71 à $3,10 \%)$. Elles se caractérisent principalement par leur hypermicrolithisme. Il s'agit essentiellement de fragments mésiaux (dont une clé de la détermination a été fournie par D. Buisson (1)). Les débris distaux et proximaux sont très rares, ainsi que les pièces entières, au demeurant exceptionnelles (un seul exemplaire, dont la morphologie, plus massive, tranche nettement avec le reste de l'ensemble).
Enfin, une pièce à dos croisé et retouche complémentaire appointante, cassée, a été classée dans la catégorie «pointe de la Gravette atypique ».

- Les lames retouchées sont nombreuses $(4,87$ à $10,32 \%$ ) et présentent une grande variabilité liée au mode, à l'ampleur et à l'obliquité de cette régularisation. Une étude factorielle des attributs a été effectuée par nous-mêmes et a permis de définir les associations de caractères les plus fréquentes. Le style technologique de la retouche « Protomagdalénienne »a été précisé par comparaison avec le site de Laugerie-Haute Est, puis replacé dans le cadre général du Paléolithique Supérieur (Aurignacien, Périgordien et Solutréen).

- Les pièces esquillées constituent l'une des caractéristiques particulières de ce gisement puisqu'on les rencontre dans des pourcentages allant de 2,65 à 6,67\%. En effet, jamais dans le Périgordien supérieur, et a fortiori dans le Protomagdalénien, une telle fréquence n'avait été observée. On les connaît dans l'Aurignacien de Corrèze alors qu'elles sont absentes de Dordogne (Sonneville-Bordes, 1960 et renseignement oral). Il s'agirait en conséquence d'un caractère régional original. Au Blot, elles sont assez typiques : les esquilles sont fréquemment unifaciales, plutôt inverses que directes d'ailleurs. Quelques-unes portent une retouche bifaciale associée à un grignotage des arêtes et prennent parfois en écharpe la totalité du support pour donner un pseudo-coup de burin.

— Les outils « archaïques », peu nombreux $(1,99$ à $3,56 \%)$, sont principalement constitués d'encoches et denticulés de divers types. Cette série est complétée par de rares racloirs et raclettes.

- Les lamelles à dos, plus généralement l'ensemble des microlithes, constituent le groupe typologique le plus important numériquement (de 55,78 à 70,80\%). Il est en majorité composé de fragments mésiaux de petites pièces à dos indéterminées parmi lesquelles existent peut-être quelques restes non identifiables de vraies microgravettes. $\mathrm{La}$ retouche croisée est très fréquente, surtout dans les niveaux les plus anciens. Notre attention s'est portée sur de petits débris à cran (proximaux ou distaux) dont l'extrémité est intacte. Suivant la théorie de fabrication proposée successivement par

(1) Suivant le système utilisé par D. Buisson pour les séries périgordiennes du Blot, une pièce à dos sera comptée comme « microgravette » si elle possède à la fois toutes les caractéristiques suivantes :

- Fragment proximal ou distal, ou pièce entière,

- Retouche croisée du dos, plane.

- Retouche complémentaire opposée appointante, ou inverse

Dans tous les autres cas, le fragment sera considéré comme lamelle à dos. 
H. Delporte puis R.B. Clay, il s'agirait de déchets de façonnage fracturés après abattage du dos (Clay, 1968).

\section{II.2. La Variabilité Typologique Interne $d u$ Protomagdalénien du Blot}

Pour chaque indice typologique préalablement déterminé, nous avons calculé la valeur moyenne théorique sur le site ainsi que les bornes de l'intervalle de variation : l'indice de dispersion étant le rapport de la seconde valeur à la première. Les résultats de cette étude statistique sont consignés dans le tableau 3.

Certains indices ne varient guère. C'est le cas des diverses catégories de burins, très stables dans la stratigraphie, et des microlithes. Dans une moindre mesure, les outils divers, les lames retouchées et les pièces esquillées montrent des variations quantitatives relatives assez modérées.

Par contre, l'indice de dispersion élevé des grattoirs s'explique ainsi. Leur faible représentation dans l'absolu $(0,55$ à 1,42\%) implique des variations relatives fortes résultant plus d'un processus aléatoire dû à l'échantillonnage que d'une réalité archéologique. D'autre part, il est intéressant de remarquer la forte valeur associée aux gravettes et microgravettes. Ce coefficient montre la perte progressive de la tradition périgordienne au cours du Protomagdalénien. Une observation similaire avait été effectuée par F. Bordes à Laugerie-Haute Est (Bordes, 1978 ; Bordes et Sonneville-Bordes, 1966).

Tout ceci confirme donc l'homogénéité des niveaux industriels du Protomagdalénien du Blot, et nous autorise à proposer le schéma évolutif suivant (de bas en haut dans la stratigraphie) :

- Extrême rareté des grattoirs, montrant des variations aléatoires de leur fréquence absolue, sans changement typologique et technique notoire.
- Augmentation de l'indice de burins, principalement des burins dièdres : les burins sur troncature retouchée ne présentant pas de variations monotones. Les burins multiples, surtout dièdres, conservent une fréquence relativement importante, peutêtre anormalement élevée dans l'ensemble 1. Par ailleurs, les burins multiples sur troncature ou mixtes apparaissent plus nombreux dans les niveaux anciens. Enfin, l'apparition et la disparition aléatoire des burins de Noailles et du Raysse ne peut être reliée avec précision à une quelconque évolution chronologique.

- Diminution continue et progressive des microgravettes, en rapport sans doute avec celle des microlithes et de la retouche croisée des dos.

- Développement important des lames retouchées, surtout sur deux bords. Substitution progressive des retouches écailleuses, abruptes et profondes par une reprise sommaire "élargie », plate ou oblique et marginale (Bosselin, 1990 à paraître).

- Croissance puis décroissance des pièces esquillées, assez typiques et fréquemment à esquilles inverses.

- Très légère augmentation des outils « archaïques », principalement des encoches et denticulés.

- Très nette diminution des microlithes, principalement pour les lamelles à dos simples (à retouche croisée), avec substitution progressive des lamelles à dos tronquées par les lamelles à dos denticulées : l'ensemble subissant d'ailleurs une forte décroissance dans le même temps. Notons enfin la perduration des fragments à cran.

III. LE PROTOMAGDALÉNIEN DU BLOT DANS SON CONTEXTE RÉGIONAL

Si l'Auvergne est une région extrêmement occupée lors des phases récentes du Magdalénien, les

\begin{tabular}{|c|c|c|c|c|c|c|c|c|c|c|c|c|c|}
\hline & IG & IOc & IP & I B & IBd & IBt & IPD & IT & ILR & ODv. & Esq. & Ild & $0 / 1 d$ \\
\hline $\begin{array}{l}\text { Le Blot, Niv. } 1 \\
\text { Le Blot, Niv. } 2 \\
\text { Le Blot, Niv. } 3 \\
\text { Le Blot, Niv. } 4\end{array}$ & $\begin{array}{l}1,42 \\
0.59 \\
0.73 \\
0,66\end{array}$ & $\begin{array}{l}1,42 \\
0.59 \\
0.18 \\
0,88\end{array}$ & $\begin{array}{l}1,78 \\
1.57 \\
1.09 \\
1,33\end{array}$ & $\begin{array}{l}16,01 \\
16.08 \\
15.30 \\
11.73\end{array}$ & $\begin{array}{r}9,60 \\
10.39 \\
9.47 \\
7,30\end{array}$ & $\begin{array}{l}4,98 \\
3,92 \\
4,37 \\
3,10\end{array}$ & $\begin{array}{l}0,71 \\
1,18 \\
3,10 \\
2,88\end{array}$ & $\begin{array}{l}2,14 \\
1.76 \\
1.46 \\
1.55\end{array}$ & $\begin{array}{r}11,74 \\
10.20 \\
8,38 \\
5,09\end{array}$ & $\begin{array}{l}3,56 \\
2,94 \\
2,19 \\
1,99\end{array}$ & $\begin{array}{l}3,91 \\
6,67 \\
5,47 \\
2,65\end{array}$ & $\begin{array}{l}50.53 \\
52.75 \\
56.28 \\
60.62\end{array}$ & $\begin{array}{r}5.34 \\
5.10 \\
5.83 \\
10.18\end{array}$ \\
\hline $\begin{array}{l}\text { Moyenne } \\
\text { Borne + } \\
\text { Borne - } \\
\text { Amplitude } \\
\text { Dispersion \% }\end{array}$ & $\begin{array}{r}0,85 \\
1,42 \\
0,59 \\
0,83 \\
98\end{array}$ & $\begin{array}{l}0,77 \\
1,42 \\
0,18 \\
1,24 \\
161\end{array}$ & $\begin{array}{r}1,44 \\
1,09 \\
1,78 \\
0,69 \\
48\end{array}$ & $\begin{array}{r}14,78 \\
16,08 \\
11,73 \\
4,35 \\
29\end{array}$ & $\begin{array}{r}9,19 \\
10,69 \\
7,30 \\
3,09 \\
34\end{array}$ & $\begin{array}{r}4,09 \\
4,98 \\
3,10 \\
1,88 \\
46\end{array}$ & $\begin{array}{r}1,97 \\
3,10 \\
0,71 \\
2,39 \\
121\end{array}$ & $\begin{array}{r}1,73 \\
2,14 \\
1,46 \\
0,68 \\
39\end{array}$ & $\begin{array}{r}8,85 \\
11,74 \\
5,09 \\
6,65 \\
75\end{array}$ & $\begin{array}{r}2,67 \\
3,56 \\
1,99 \\
1,57 \\
59\end{array}$ & $\begin{array}{r}4,68 \\
6,67 \\
2,65 \\
4,02 \\
86\end{array}$ & $\begin{array}{r}55,05 \\
60,62 \\
50,53 \\
10,09 \\
18\end{array}$ & $\begin{array}{r}6.61 \\
10.18 \\
5.10 \\
5.08 \\
77\end{array}$ \\
\hline
\end{tabular}


vestiges industriels du Gravettien restent encore trop rares pour une diagnose régionale précise. Ainsi, mis à part les niveaux périgordiens du Blot, les deux seuls sites actuellement reconnus sont la grotte de Tatevin à Chanteuges et l'abri du Rond à Saint-Arcons-d'Allier.

Le premier, fouillé successivement par P. de Brun à la fin du XIX ${ }^{\mathrm{e}}$ siècle, P. Bout puis J. Virmont livre une série lithique assez pauvre (49 outils). Les caractères typologiques sont les suivants : fréquence moyenne des grattoirs ( $\mathrm{IG}=18,4 \%)$, surtout sur bout de lame, dépassant légèrement les burins (IB = $16,3 \%$ ) parmi lesquels les dièdres dominent les exemplaires sur troncature retouchée (IBd $=10,2 \%$ et $\mathrm{IBt}=4,1 \%)$. Les gravettes sont absentes mais deux fragments de microgravette ont été retrouvés. Les lames retouchées, à retouche écailleuse, sont rares $(2,0 \%)$, alors que les encoches et denticulés $(10,2 \%)$ et les microlithes, à retouche principalement croisée $(24,5 \%)$ abondent. Notons également la présence de deux pièces esquillées.

L'abri du Rond à Saint-Arcons est également très pauvre (50 outils). Les grattoirs, surtout du type aurignacien, dominent largement les burins ( $\mathrm{IG}=$ $36,0 \%$ et $\mathrm{IB}=30,0 \%$ ). Les burins dièdres sont plus nombreux que les burins sur troncature retouchée $(\mathrm{IBd}=14,0 \%$ et $\mathrm{IBt}=10,0 \%):$ certains évoquant morphologiquement et stylistiquement leurs homologues du Protomagdalénien du Blot. Les gravettes sont absentes et les lames retouchées, à retouche écailleuse, grignotée ou parfois plate «élargie » (comme les burins), sont abondantes $(12,0 \%)$. Notons enfin une pièce à gibbosité, proche de celles rencontrées au Cirque de la Patrie et la relative faiblesse des microlithes $(2,0 \%)$.

Les niveaux périgordiens du Blot sont actuellement en cours d'étude au Musée des Antiquités Nationales par D. Buisson. Toutefois, grâce à son extrême obligeance, nous avons eu accès aux collections qui, pour les deux ensembles reconnus, présentent les caractères suivants : absence des grattoirs et des perçoirs, développement limité des burins $(10-15 \%)$, parmi lesquels on note une inversion du rapport $\mathrm{Bd} / \mathrm{Bt}$ du niveau le plus ancien vers le plus récent, rareté des lames retouchées, abondance des burins multiples mixtes, des microgravettes $(25-30 \%)$, et des microlithes, principalement à retouche croisée du dos (45-50 \%).

La faible représentation du Périgordien supérieur en Auvergne rend difficile, en l'état actuel des connaissances, un travail de synthèse sur le cycle gravettien de la région. C'est ainsi que les trois sites répertoriés présentent tous des particularités plus ou moins marquées. Le Protomagdalénien du Blot apparaît alors comme un ensemble original révélant ponctuellement des convergences avec les sites de
Tatevin et du Rond : supériorité des burins dièdres sur les burins sur troncature retouchée, abondance des microlithes, à retouche croisée du dos et présence de pièces esquillées (Tatevin), des lames retouchées, à retouche écailleuse (Le Rond). A l'inverse, des caractères discriminants empêchent un rapprochement strict entre ces trois gisements : indice des grattoirs supérieur à celui des burins (Tatevin, Le Rond), absence de microgravette et des microlithes (Le Rond). Au contraire, les points communs entre Périgordien et Protomagdalénien du Blot apparaissent nettement : fréquence des microgravettes et des microlithes, à retouche croisée, rareté des grattoirs et domination des burins dièdres sur les exemplaires sur troncature retouchée (fig. 1).

Devant la faiblesse des sites reconnus actuellement, il est difficile de déterminer l'origine du peuplement périgordien de l'Auvergne. Des éléments typologiques plaident en faveur de contacts réguliers avec le Bassin Parisien (pièce gibbeuse au Rond) tandis que la fréquence des microlithes et des microgravettes nous incite à nous tourner vers des industries plus méridionales (faciès « Rhodanien » de J. Combier ou même Gravettien de la Provence) (Combier, 1967 ; Onoratini, 1974, 1979). Enfin, et nous le verrons dans le chapitre qui va suivre, c'est en Périgord (principalement pour les périodes finales) qu'il faut reconnaître les affinités les plus satisfaisantes.

IV. LE PROTOMAGDALENIEN DU BLOT DANS SON CONTEXTE CULTUREL

En dehors de Laugerie-Haute, en Dordogne, la culture matérielle du Périgordien VI et VII était, à la fin des années 1960, très mal connue. Depuis, plusieurs sites stratifiés ont livré des niveaux industriels attribuables à cette période : Corbiac (fouilles F. Bordes), Le Roc de Combe (fouilles F. Bordes et J. Labrot), Rabier (fouilles J. Guichard), l'abri Pataud (fouilles H.L. Movius) et le Flageolet I (fouilles J.-Ph. Rigaud). Si les gisements de Rabier et du Roc de Combe ne sont pas publiés, les indications données par D. Peyrony puis complétées par F. Bordes pour Laugerie-Haute ainsi que celles, toujours incomplètes, de Corbiac et de l'abri Pataud permettent déjà une première diagnose de la fin du cycle gravettien (Bordes, 1958, 1968, 1978 ; Bordes et Labrot, 1967 ; Movius, 1975 ; Bricker et David, 1984 ; Peyrony, 1938 ; Rigaud, 1982).

\section{IV.1. Le Périgordien VI}

Le Périgordien « III », découvert à LaugerieHaute (côtés Est et Ouest), par D. Peyrony 


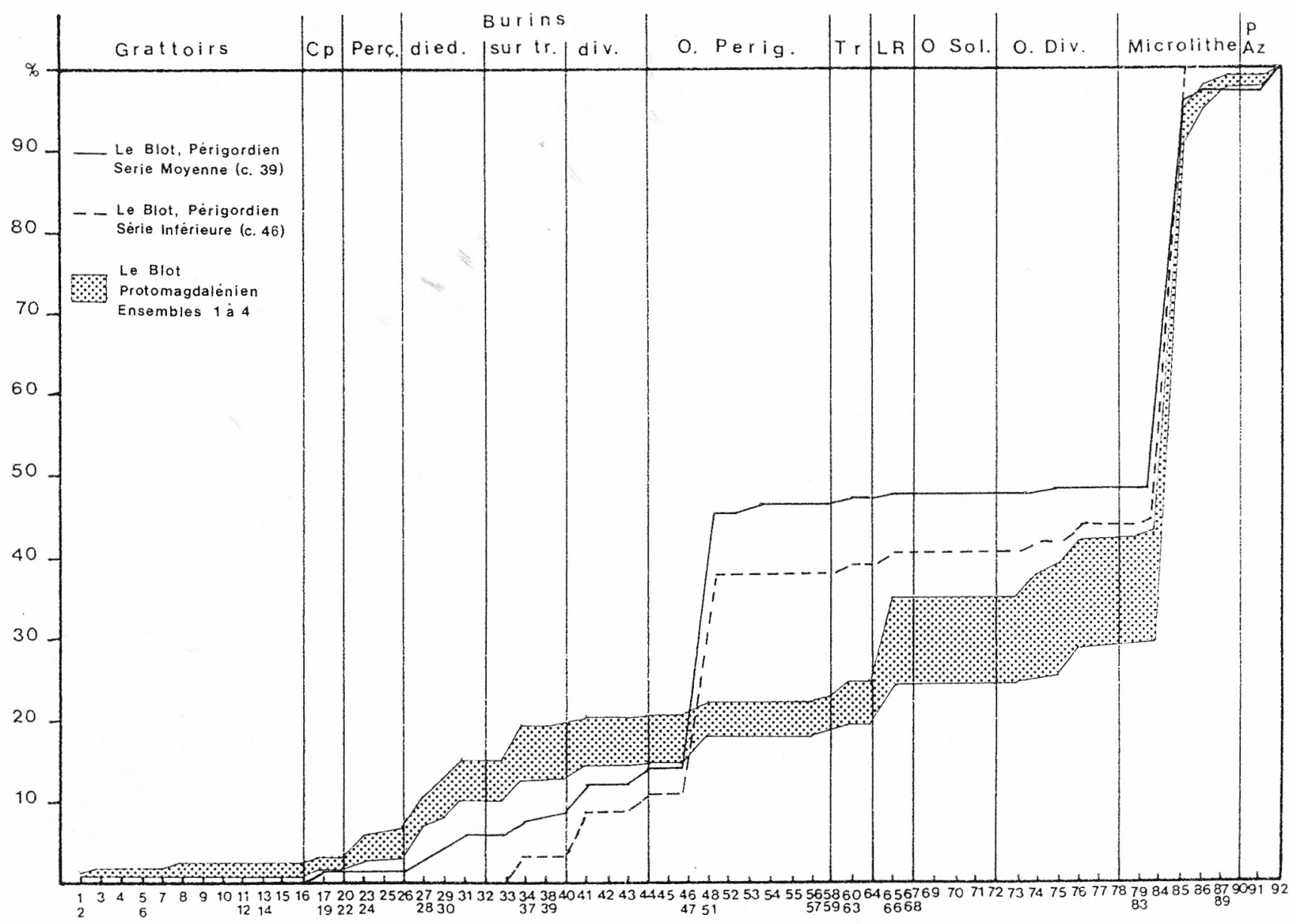

Fig. 1 - Diagrammes cumulatifs comparés des industries lithiques du Protomagdalénien et du Périgordien Supérieur du Blot (d'après D. Buisson inédit).

(couches B et B') a été reconsidéré sur des bases nouvelles suite aux fouilles de F. Bordes. Se basant sur une stratigraphie fine et des études sédimentologiques (H. Laville), il a été établi qu'il devait être contemporain, voire légèrement plus récent que le Périgordien V à burins de Noailles. Il devint donc logique que le Périgordien «III » devait être rebaptisé Périgordien VI (Bordes, 1978 ; Laville, 1964, 1975 ; Peyrony, 1938 ; Sonneville-Bordes, 1960). L'industrie lithique de Laugerie-Haute a été décrite dans le détail par D. de Sonneville-Bordes. Elle se caractérise (resp. couches B et B') par l'abondance des burins ( $\mathrm{IB}=22,1$ et $32,4 \%$ ) sur troncature retouchée, souvent triples ou quadruples (IBt $=11,5$ et $15,2 \%)$, par la rareté des burins dièdres (IBd = 5,9 et $8,2 \%$ ), par l'absence de la variété des grattoirs, presque toujours simples ( $\mathrm{IG}=14,2$ et $9,5 \%$ ). Les pointes de la Gravette, peu nombreuses dans les niveaux inférieurs $(1,7 \%)$, progressent dans les niveaux supérieurs $(8,7 \%)$ : les microgravettes achevant de donner à cet ensemble une allure fran- chement périgordienne. Les outils spéciaux du Périgordien Vc sont absents, à l'exception de quelques Noailles atypiques. Les séries sont complétées par des lamelles à dos $(19,9$ et $25,5 \%)$, des lames tronquées $(10,6$ et $7,3 \%)$ et surtout des encoches-denticulés (26,1 et $14,9 \%$ ) (Peyrony, 1938, p. 14 ; Sonneville-Bordes, 1958).

L'industrie lithique du Périgordien VI de l'abri Pataud possède, d'après les rares publications le concernant, un équilibre typologique proche de son homologue de Laugerie-Haute : supériorité des burins ( $\mathrm{IB}=31,4 \%)$ sur les grattoirs $(\mathrm{IG}=13,8 \%)$, des burins sur troncature retouchée $(\mathrm{IBt}=18,3 \%)$ sur les burins dièdres (IBd $=7,3 \%)$, abondance des pointes de la Gravette $(6,4 \%)$ et surtout des microgravettes $(14,8 \%)$, fréquence moyenne des lames tronquées $(4,89 \%)$, des lamelles à dos $(1,1 \%)$ et prolifération des encoches $(13,4 \%)$. Malgré quelques dissemblances, les études connexes groupant la sédimentologie, la palynologie et la paléon- 
tologie s'accordent pour attribuer les deux séries à une même phase climatique relativement froide (Farrand, 1975 ; Laville, 1975).

L'industrie de l'ensemble I-III du Flageolet I a été étudiée par J.-Ph. Rigaud et apparait contemporaine du Périgordien VI de Laugerie-Haute. Elle se caractérise par la rareté en grattoirs ( $\mathrm{IG}=11,5 \%)$, largement dépassés par les burins ( $\mathrm{IB}=31,6 \%$ ) parmi lesquels les exemplaires sur troncature dominent également les dièdres (IBt $=16,1 \%$ et IBd $=$ $13,8 \%$ ). Les gravettes et microgravettes sont présentes, quoique assez rares $(5,2 \%)$ tandis qu'on remarque l'abondance des encoches et denticulés $(13,2 \%)$, des lames tronquées $(9,8 \%)$, et surtout des lames retouchées (14,4\%) (Rigaud, 1982). La couche $1 \mathrm{du}$ Roc de Combe semble légèrement antérieure au niveau I-III du Flageolet et contient, d'après les indications sommaires fournies par F. Bordes, un pourcentage important de pointes à dos (Bordes et Labrot, 1967).

A Rabier, près de Bergerac, une industrie évoluée, à nombreuses gravettes et microgravettes, a été mise au jour par J. Guichard. Une première approche technologique a été effectuée par A. Morala (Morala, 1987).

Le Périgordien VI se retrouve également dans le Bassin Parisien au Cirque de la Patrie dans un faciès à burins sur troncature retouchée $(\mathrm{IBt}=22,6 \%)$ et pointes de la Gravette $(25,0 \%)$. L'industrie lithique se caractérise par la faiblesse des grattoirs $(\mathrm{IG}=$ $6,6 \%$ ), nettement dominés par les burins (IB = $31,0 \%)$, dont de rares dièdres ( $\mathrm{IBd}=3,6 \%)$. La série est complétée par des lames tronquées $(19,1 \%)$, des lamelles à dos $(3,0 \%)$, quelques pièces gibbeuses $(4,1 \%)$ (Schmider, 1971).

\section{IV.2. Le Périgordien évolué}

Le site de Corbiac, près de Bergerac, a livré plusieurs niveaux très proches du Périgordien VI. Dans les publications préliminaires (resp. couches 1 et 1A), F. Bordes insiste sur la relative rareté des grattoirs $(\mathrm{IG}=7,0$ et $6,4 \%)$, surtout sur lame non retouchée, la supériorité des burins dièdres $(\mathrm{IBd}=$ 26,4 et $20,8 \%$ ), sur les burins sur troncature retouchée (IBt $=10,6$ et 8,1\%), l'absence des Noailles et la présence significative des gravettes et microgravettes $(11,7$ et 10,2 \%), de pièces à soie et à cran, de lames retouchées $(6,2$ et $6,1 \%)$ et de lamelles à dos (2,9 et $4,4 \%)$. L'outillage est complété par des encoches et denticulés $(12,5$ et $13,6 \%)$ et des lames tronquées (7,0 et 8,7\%) (Bordes, 1968). L'industrie de Corbiac apparaît un peu plus évoluée (récente ?) que celle de Laugerie-Haute et se distingue de cette dernière par l'inversion du rapport IBd/IBt. Au contraire, les ressemblances morphologiques et stylistiques plaident en faveur d'une évolution depuis le Périgordien VI (vers le Protomagdalénien) (Bordes et Sonneville-Bordes, 1966).

En dehors de la région classique du Périgord, la connaissance s'est accrue grâce aux découvertes du Roc de Cavart et de Fresquet en Agenais, des Vachons en Charente et des sites de Baume Périgaux et de la Verrière, respectivement en Provence et en Languedoc oriental.

Le site de Cavart, fouillé par L. Coulonges vers 1935, a été étudié par J.-M. Le Tensorer (Le Tensorer, 1981). Le niveau industriel gravettien est intermédiaire entre les séries de Laugerie et de Corbiac : indice de grattoirs très bas $(\mathrm{IG}=6,0 \%)$, presque toujours simples, dépassés par les burins $(\mathrm{IB}=30,6 \%)$, les types dièdres et sur troncature retouchée s'équilibrant $(\mathrm{IBd}=\mathrm{IBt}=11,5 \%)$, rareté des burins de Noailles (moins de $1 \%$ ), abondance des pointes de la Gravette et des microgravettes $(17,1 \%)$, des lames tronquées $(7,9 \%)$, fréquence moyenne des encoches et denticulés $(6,4 \%)$, des lames retouchées $(2,0 \%)$ et des microlithes $(5,2 \%)$. La série de surface de Fresquet (Lot-et-Garonne), montre une répartition typologique plus proche de celle de Corbiac : peu de grattoirs ( $\mathrm{IG}=3,4 \%$ ), burins dominant ( $\mathrm{IB}=32,60 \%$ ) parmi lesquels les dièdres sont les plus nombreux (IBd $=13,6 \%$ et $\mathrm{IBt}=7,1 \%$ ), présence de Noailles $(7,8 \%)$, développement des microgravettes $(10,5 \%)$, des lames tronquées $(15,1 \%)$ et des encoches-denticulés $(13,9 \%)$ (Morala, 1984).

Le site des Vachons à Voulgézac (Charente) a été fouillé successivement par J. Bouyssonie et J. Coiffard puis publié par J. Bouyssonie et $\mathrm{D}$. de Sonneville-Bordes (Bouyssonie et SonnevilleBordes, 1956). L'industrie présente un équilibre typologique proche des niveaux archéologiques précédents : supériorité des burins ( $\mathrm{IB}=57,8$ à $32,2 \%$ ) sur les grattoirs $(\mathrm{IG}=16,3$ à $13,6 \%)$, des burins dièdres $(\mathrm{IBd}=30,7$ à $16,3 \%)$ sur les burins sur troncature retouchée $(\mathrm{IBt}=16,0$ à $7,3 \%)$, présence de quelques gravettes et microgravettes (1,2 à $5,7 \%)$, pièces à cran, nombreuses troncatures (IT = $4,5$ à $11,8 \%)$ et encoches-denticulés $(4,9$ à $10,0 \%)$. Les lames retouchées sont rares $(3,4$ à $3,7 \%)$ et les microlithes absents.

L'habitat de la Baume Périgaux est décrit par G. Onoratini (Onoratini, 1982) : extrême rareté des grattoirs ( $\mathrm{IG}=2,2 \%)$, développement moyen des burins ( $\mathrm{IB}=12,2 \%)$, dont principalement des dièdres ( $\mathrm{IBd}=7,8 \%, \mathrm{IBt}=3,3 \%)$, abondance des gravettes et microgravettes $(6,7$ et $25,6 \%)$, des lamelles à dos $(34,4 \%)$. 
Le gisement de plein air de la Verrière, dans le département du Gard, a été publié par F. Bazile (Bazile, 1981). Les grattoirs ( $\mathrm{IG}=10,2 \%$ ) sont fréquemment épais, et dépassés par les burins ( $\mathrm{IB}=$ $26,3 \%)$ : les dièdres étant légèrement inférieurs aux exemplaires sur troncature retouchée (IBd $=10,2 \%$ et $\mathrm{IBt}=12,9 \%)$. Là encore, gravettes $(9,8 \%)$ et microgravettes $(4,4 \%)$ abondent. Les lamelles à dos $(2,2 \%)$, les lames tronquées $(2,7 \%)$ et les encoches $(8,7 \%)$ sont moyennement représentées. L'industrie est complétée par de nombreuses lames retouchées $(13,1 \%)$ évoquant parfois morphologiquement les pointes à face plane du Midi de la France (pointes Aréniennes).

Les industries du Périgordien final voient leur équilibre typologique se modifier quelque peu dans le temps. Après une phase ancienne qui rappelle le Périgordien IV, l'évolution montre un enrichissement en gravettes et microgravettes, en burins dièdres (au détriment des burins sur troncature retouchée), et en lames retouchées et voit la raréfaction progressive des encoches et denticulés et des lames tronquées pour annoncer clairement le Protomagdalénien.

\section{IV.3. Le Protomagdalénien}

Découvert par D. Peyrony au-dessus du Périgordien «III », le Protomagdalénien de la couche F est décrit tout d'abord comme «un mélange de l'Aurignacien et du Périgordien évoluant vers des formes nouvelles à tendance magdalénienne » (Peyrony, 1938). Plus tard, il le considère comme terminant le cycle Périgordien, sans rapport direct avec la civilisation aurignacienne, en accord avec D. de Sonneville-Bordes et F. Bordes (Peyrony, 1952 ; Bordes et Sonneville-Bordes, 1966). Les nouvelles recherches de F. Bordes à Laugerie-Haute ont confirmé l'existence, sous le Protomagdalénien proprement dit (c. 36), d'un niveau, plus pauvre, mais contenant à la fois des caractères archaïques et évolués. D'après l'auteur, il pourrait s'agir d'un faciès de passage entre Périgordien VI et VII. Les caractéristiques typologiques du Protomagdalénien (couche 36) ont été exposées par F. Bordes : pauvreté en grattoirs $(\mathrm{IG}=$ $7,3 \%$ ), surtout sur bout de lames non retouchées, richesse en burins ( $\mathrm{IB}=31,5 \%$ ) principalement dièdres, comme à Corbiac (IBd $=22,3 \%$ et IBt $=$ $6,5 \%$ ). Les burins multiples sont nombreux et les gravettes et microgravettes pratiquement absentes. Les lamelles à dos sont abondantes et fréquemment tronquées $(21,4 \%)$. La série est complétée par quelques outils composites, de rares perçoirs et becs, et une proportion moyenne d'outils « archaïques », principalement d'encoches et denti- culés $(9,5 \%)$ et des lames retouchées $(8,0 \%)$ ou tronquées $(5,8 \%)$. Il n'y a pas de pièces esquillées (Bordes, 1978). Une datation radiocarbone a été produite (GrN-1876), $21980 \pm 250 \mathrm{BP}$ (couche 36).

Cette industrie se rattache très nettement au cycle gravettien et justifie pleinement la dénomination de Périgordien VII, malgré quelques caractères propres. En particulier, il apparaît une nette filiation entre le Périgordien final tel qu'on le rencontre à Corbiac ou au Roc de Combe (même supériorité des burins sur les grattoirs, des burins dièdres sur ceux sur troncature retouchée, existence d'une retouche latérale proche de celle du Protomagdalénien). $\mathrm{Au}$ contraire, il semble exister une coupure typologique marquée entre les Périgordien VI et VII de Laugerie-Haute (Bordes et Sonneville-Bordes, 1966).

Le Protomagdalénien a ensuite été retrouvé à l'abri Pataud par H.L. Movius. Bien que cette industrie ne soit que très partiellement publiée, les recherches de R.B. Clay mettent en évidence une certaine parenté entre les deux sites de Dordogne (Clay, 1968).

Des datations au radiocarbone situent plus précisément les positions respectives des Périgordien VI et VII de Pataud:

- Périgordien VI : 3 dates entre 23000 et $21500 \mathrm{BP}$.

- Périgordien VII : 10 dates entre 21940 et 19120 BP (avec plus de la moitié d'entre elles situées dans la plage 21380-20340 BP).

Une industrie évoluée a été retrouvée par F. Champagne aux Fieux à Miers (Champagne et Jaubert, 1979). Les grattoirs, très rares, sont dominés par les burins parmi lesquels les exemplaires sur troncature retouchée sont légèrement plus nombreux que les dièdres. La série est complétée par près de $55 \%$ de microlithes divers (lamelles à dos principalement) et $5 \%$ de lames retouchées. Certains auteurs ont rapproché ce niveau du Protomagdalénien. Nous préférons réserver notre avis en l'attente d'une étude plus complète : en particulier, le style de la retouche latérale des lames, la présence éventuelle de fragments de microgravettes parmi les microlithes (retouche d'aménagement de la pointe associée à un dos à retouche croisée sur enclume par exemple), et l'étude du contexte restant à préciser. Il en est de même du site de la Pointe des Brosses (Montigny-sur-Loing). Cette série lithique possède des caractères indubitablement magdaléniens : burins l'emportant sur les grattoirs, burins dièdres surclassant les burins sur troncature retouchée et lamelles à dos abondantes (plus de $30 \%$ ). Toutefois, deux dates C14 donnent : (OXA-179) : 
$22200 \pm 660 \mathrm{BP}$ et $(\mathrm{OXA}-180): 22500 \pm 600 \mathrm{BP}$ rendant ainsi difficile, en l'absence de repères stratigraphiques, la distinction entre Périgordien final de plein air et Magdalénien dans le Bassin Parisien (Schmider, 1971, 1983).

IV.4. Le Blot dans le contexte culturel de la fin du Gravettien

Ce rapide tour d'horizon de la culture matérielle du Périgordien VI et du Protomagdalénien met clairement en évidence une opposition entre les industries lithiques de ces deux phases. En particulier, on assiste, en remontant dans la stratigraphie, à une complète inversion du rapport $\mathrm{Bd} / \mathrm{Bt}$, une augmentation sensible des burins multiples, des lames retouchées et des microlithes. A l'inverse, les groupes typologiques des grattoirs, des burins sur troncature retouchée, des pointes et micropointes de la Gravette subissent une baisse spectaculaire, (tabl. 4).

Il est donc évident que les comparaisons culturelles avec le Protomagdalénien du Blot ne concerneront que les industries dites «évoluées » : c'est-àdire essentiellement le Protomagdalénien de Laugerie-Haute Est (de l'abri Pataud), le Périgordien de Corbiac, et, dans une moindre mesure, les sites de Fresquet, de la Pointe des Brosses et du Roc de Cavart. Nous écartons volontairement les niveaux des Vachons, de la Verrière et de la Baume Périgaux qui, pour des raisons diverses, risquent d'introduire un biais : fouilles anciennes aux Vachons, gisement de surface à la Verrière et affinités orientales pour la Baume Périgaux.

La lecture du tableau 4 amène quelques remarques d'ordre général. Si la filiation Périgordien VI-Protomagdalénien est établie depuis les travaux de F. Bordes, il existe manifestement une coupure typologique entre le Périgordien VI «classique » et le Périgordien évolué, du type Corbiac. L'évolution se traduit dans l'outillage par la décroissance des grattoirs, des burins sur troncature retouchée, des gravettes et microgravettes, des lames tronquées et des outils « archaïques », au profit des burins dièdres, des perçoirs, des lames retouchées et des microlithes. Ainsi, serions-nous enclins à nuancer la théorie de F. Bordes en proposant de voir dans les niveaux à burins dièdres une étape ultime dans le développement du Gravettien, mais ne succédant pas directement sans passage par une industrie de transition au Périgordien VI à nombreux burins sur troncature retouchée. Les sites actuellement publiés ne fournissent qu'une réponse très partielle. En effet, si l'inversion du rapport $\mathrm{Bd} / \mathrm{Bt}$ paraît l'un des indicateurs d'évolution les plus fiables, les séries disponibles (Corbiac, Fresquet, le Roc de Cavart, les Vachons, sans prendre en compte les données du Sud-Est de la France et du Bassin Parisien) conservent encore des caractères « archaïques »: troncatures, encoches-denticulés et microgravettes à Corbiac, burins de Noailles, microgravettes et encoches-denticulés à Fresquet, équilibre $\mathrm{Bd} / \mathrm{Bt}$, microgravettes au Roc de Cavart, grat-

\begin{tabular}{|c|c|c|c|c|c|c|c|c|c|c|c|}
\hline Site & Niveau & IG & IP & IB & IBd & $\mathrm{IBt}$ & IPD & IT & $\mathbf{I L R}$ & $74-75$ & Mic \\
\hline $\begin{array}{l}\text { Le Blot } \\
\text { Le Blot } \\
\text { Le Blot } \\
\text { Le Blot }\end{array}$ & $\begin{array}{l}\text { Ensemble } 1 \\
\text { Ensemble 2 } \\
\text { Ensemble } 3 \\
\text { Ensemble } 4\end{array}$ & $\begin{array}{l}1,4 \\
0,6 \\
0,7 \\
0,7\end{array}$ & $\begin{array}{l}1.8 \\
1,6 \\
1,1 \\
1,3\end{array}$ & $\begin{array}{l}16,0 \\
16,1 \\
15,3 \\
11,7\end{array}$ & $\begin{array}{r}9,6 \\
10,4 \\
9,5 \\
7,3\end{array}$ & $\begin{array}{l}5,0 \\
3,9 \\
4,4 \\
3,1\end{array}$ & $\begin{array}{l}0,7 \\
1,2 \\
3,1 \\
2,9\end{array}$ & $\begin{array}{l}2,1 \\
1,8 \\
1,5 \\
1,6\end{array}$ & $\begin{array}{r}11,7 \\
10,2 \\
8,4 \\
5,1\end{array}$ & $\begin{array}{l}3,6 \\
2,9 \\
1,8 \\
1,8\end{array}$ & $\begin{array}{l}55,9 \\
57,9 \\
62,1 \\
70,8\end{array}$ \\
\hline $\begin{array}{l}\text { Laugerie-Haute Est } \\
\text { Laugerie-Haute Est } \\
\text { Laugerie-Haute Est } \\
\text { Abri Pataud }\end{array}$ & $\begin{array}{l}\text { Couche } 36 \\
\text { Couche } 38 \\
\text { Couche F } \\
\text { Couche } 2\end{array}$ & $\begin{array}{l}7,3 \\
2,7 \\
9,7 \\
5,3\end{array}$ & $\begin{array}{l}2,7 \\
2,7 \\
3,7 \\
3,7\end{array}$ & $\begin{array}{l}31,5 \\
40,0 \\
40,7 \\
29,9\end{array}$ & $\begin{array}{l}22,3 \\
26,7 \\
27,1 \\
22,5\end{array}$ & $\begin{array}{r}6,5 \\
4,0 \\
10,3 \\
5,4\end{array}$ & $\begin{array}{l}0,0 \\
5,3 \\
2,5 \\
1,2\end{array}$ & $\begin{array}{l}5,8 \\
6,7 \\
2,4 \\
1,5\end{array}$ & $\begin{array}{l}8,0 \\
5,3 \\
6,9 \\
8,6\end{array}$ & $\begin{array}{r}9,5 \\
17,3 \\
2,4 \\
10,3\end{array}$ & $\begin{array}{r}21,4 \\
6,7 \\
21,9 \\
35,0\end{array}$ \\
\hline $\begin{array}{l}\text { Corbiac } \\
\text { Corbiac } \\
\text { La Pointe des Brosses } \\
\text { Fresquet } \\
\text { Le Roc de Cavart } \\
\text { Les Vachons I } \\
\text { Les Vachons I } \\
\text { Les Vachons II }\end{array}$ & $\begin{array}{l}\text { Couche } 1 \\
\text { Couche 1A } \\
\text { Couche } 5 \text { (JB) } \\
\text { Couche } 5 \text { (JC) } \\
\text { Couche } 5 \text { (JC) }\end{array}$ & $\begin{array}{r}7.0 \\
6.4 \\
5.9 \\
3.4 \\
6.0 \\
14.8 \\
16,3 \\
13,6\end{array}$ & $\begin{array}{l}2,2 \\
1,5 \\
1,2 \\
4,1 \\
3.2 \\
1,2 \\
1,8 \\
2,8\end{array}$ & $\begin{array}{l}41,8 \\
33,3 \\
41,8 \\
32,6 \\
30,6 \\
57.8 \\
32,6 \\
32,2\end{array}$ & $\begin{array}{l}26.4 \\
20,8 \\
32,4 \\
13,6 \\
11,5 \\
30.7 \\
16.3 \\
17,5\end{array}$ & $\begin{array}{r}10,6 \\
8,1 \\
2,4 \\
7,1 \\
11,5 \\
16,0 \\
13.6 \\
7,3\end{array}$ & $\begin{array}{r}11,7 \\
10,2 \\
0,0 \\
10.5 \\
17,1 \\
1,2 \\
1,8 \\
5,7\end{array}$ & $\begin{array}{r}7,0 \\
8,7 \\
2,4 \\
15,1 \\
7,9 \\
4,9 \\
11,8 \\
10,2\end{array}$ & $\begin{array}{l}6,2 \\
6,1 \\
2,4 \\
2,0 \\
2,0 \\
3,7 \\
3,6 \\
3,4\end{array}$ & $\begin{array}{r}12,5 \\
13,6 \\
4,1 \\
13,9 \\
6,4 \\
4,9 \\
10,0 \\
9,1\end{array}$ & $\begin{array}{r}2,9 \\
4,4 \\
32,9 \\
3,7 \\
5,2 \\
4,7 \\
0,0 \\
0,0\end{array}$ \\
\hline $\begin{array}{l}\text { Laugerie-Haute Est } \\
\text { Laugerie-Haute Est } \\
\text { Laugerie-Haute Est } \\
\text { Laugerie-Haute Ouest } \\
\text { Abri Pataud } \\
\text { Le Flageolet I } \\
\text { Le Cirque de la Patrie }\end{array}$ & $\begin{array}{l}\text { Couche B } \\
\text { Couche B' } \\
\text { Couche BB' } \\
\text { Couche B } \\
\text { Couche } 3 \\
\text { Couche I-III }\end{array}$ & $\begin{array}{r}14,2 \\
9,5 \\
27,1 \\
34,1 \\
13,8 \\
11,5 \\
6,6\end{array}$ & $\begin{array}{l}1,2 \\
0,0 \\
0,0 \\
0,4 \\
1,8 \\
0,6 \\
1,2\end{array}$ & $\begin{array}{l}22,1 \\
32,4 \\
33,6 \\
31,4 \\
31,4 \\
31,6 \\
31,0\end{array}$ & $\begin{array}{r}5,9 \\
8,2 \\
7,4 \\
7,7 \\
7,3 \\
13,8 \\
3,6\end{array}$ & $\begin{array}{l}11,5 \\
15,2 \\
24,5 \\
17,8 \\
18,3 \\
16,1 \\
22,6\end{array}$ & $\begin{array}{r}1,7 \\
0,7 \\
0,3 \\
1,8 \\
20,3 \\
5,2 \\
25,0\end{array}$ & $\begin{array}{r}10,6 \\
7,3 \\
0,6 \\
5,4 \\
4,9 \\
9,8 \\
19,1\end{array}$ & $\begin{array}{r}0,2 \\
0,0 \\
0,0 \\
1,1 \\
2,4 \\
14,4 \\
0,0\end{array}$ & $\begin{array}{r}26,1 \\
14,9 \\
2,4 \\
12,4 \\
14,8 \\
13,2 \\
0,0\end{array}$ & $\begin{array}{r}19,9 \\
25,9 \\
30,4 \\
7,9 \\
1,1 \\
10,3 \\
3,0\end{array}$ \\
\hline $\begin{array}{l}\text { LE BLOT PROTOMAGDALENIEN } \\
\text { PROTOMAGDALENIEN } \\
\text { PERIGORDIEN EVOLUE } \\
\text { PERIGORDIEN VI }\end{array}$ & $\begin{array}{l}\text { Moyenne } \\
\text { Moyenne } \\
\text { Moyenne } \\
\text { Moyenne }\end{array}$ & $\begin{array}{r}0,9 \\
6,3 \\
9,2 \\
16,7\end{array}$ & $\begin{array}{l}1,5 \\
3,2 \\
2,3 \\
0,7\end{array}$ & $\begin{array}{l}14,8 \\
35,5 \\
37,8 \\
30.5\end{array}$ & $\begin{array}{r}9,2 \\
24,7 \\
21,2 \\
7,7\end{array}$ & $\begin{array}{r}4,1 \\
6,6 \\
9,6 \\
18,0\end{array}$ & $\begin{array}{l}2,0 \\
2,3 \\
7,3 \\
7,9\end{array}$ & $\begin{array}{l}1,8 \\
4,1 \\
8,5 \\
8.2\end{array}$ & $\begin{array}{l}8,9 \\
7,2 \\
3,7 \\
2,6\end{array}$ & $\begin{array}{r}2.5 \\
9,9 \\
9.3 \\
12.0\end{array}$ & $\begin{array}{r}61.7 \\
21.3 \\
6.7 \\
14.0\end{array}$ \\
\hline
\end{tabular}


toirs, burins sur troncature retouchée, lames tronquées aux Vachons. D'autre part, la croissance des lames retouchées ne paraît pas totalement synchrone du phénomène précédent, à la lueur des données de Corbiac, Fresquet, Cavart et les Vachons principalement. Il faudra donc peut-être rechercher ailleurs une hypothétique descendance.

Le site du Blot, à Cerzat, se situe parfaitement au sein de la culture protomagdalénienne. Si les études sédimentologiques militent en faveur d'une attribution chronologique plus récente qu'en Dordogne (phase climatique tempérée et peu humide, vraisemblablement interstadiaire), l'équilibre typologique est, malgré quelques disparités flagrantes, sensiblement comparable à celui de Laugerie-Haute Est (de Pataud) et de Corbiac (fig. 2). A savoir : extrême rareté des grattoirs, surtout simples, faible développement des outils composites et des perçoirs, importance numérique des burins, principalement dièdres, et souvent multiples, absence des burins de Noailles, présence relativement faible des microgravettes, lames retouchées assez nombreuses. Toutefois, deux caractères discriminants empêchent un strict rapprochement : le foisonnement des microlithes et la quantité non négligeable de pièces esquillées au Blot. Dans une moindre mesure, les encoches-denticulés, les lames tronquées, les burins (Bd, Bt, Bd multiples, B. multiples mixtes), et les grattoirs sont légèrement mieux représentés en Dordogne. Comme nous l'avons exposé dans un chapitre antérieur, les pièces esquillées constituent, comme dans l'Aurignacien de Corrèze, un facteur régional (elles se rencontrent également dans le Périgordien supérieur d'Auvergne). Par contre, l'abondance des microlithes apparaît plus surprenante, sans doute liée à une spécificité locale dont il faudra sans doute négliger la portée « culturelle».

Pour effectuer des comparaisons plus pertinentes, nous avons été amenés à « délamelliser» les séries, c'est-à-dire à exclure du décompte les numéros 84 à 90 de la liste-type, ainsi que les pièces esquillées

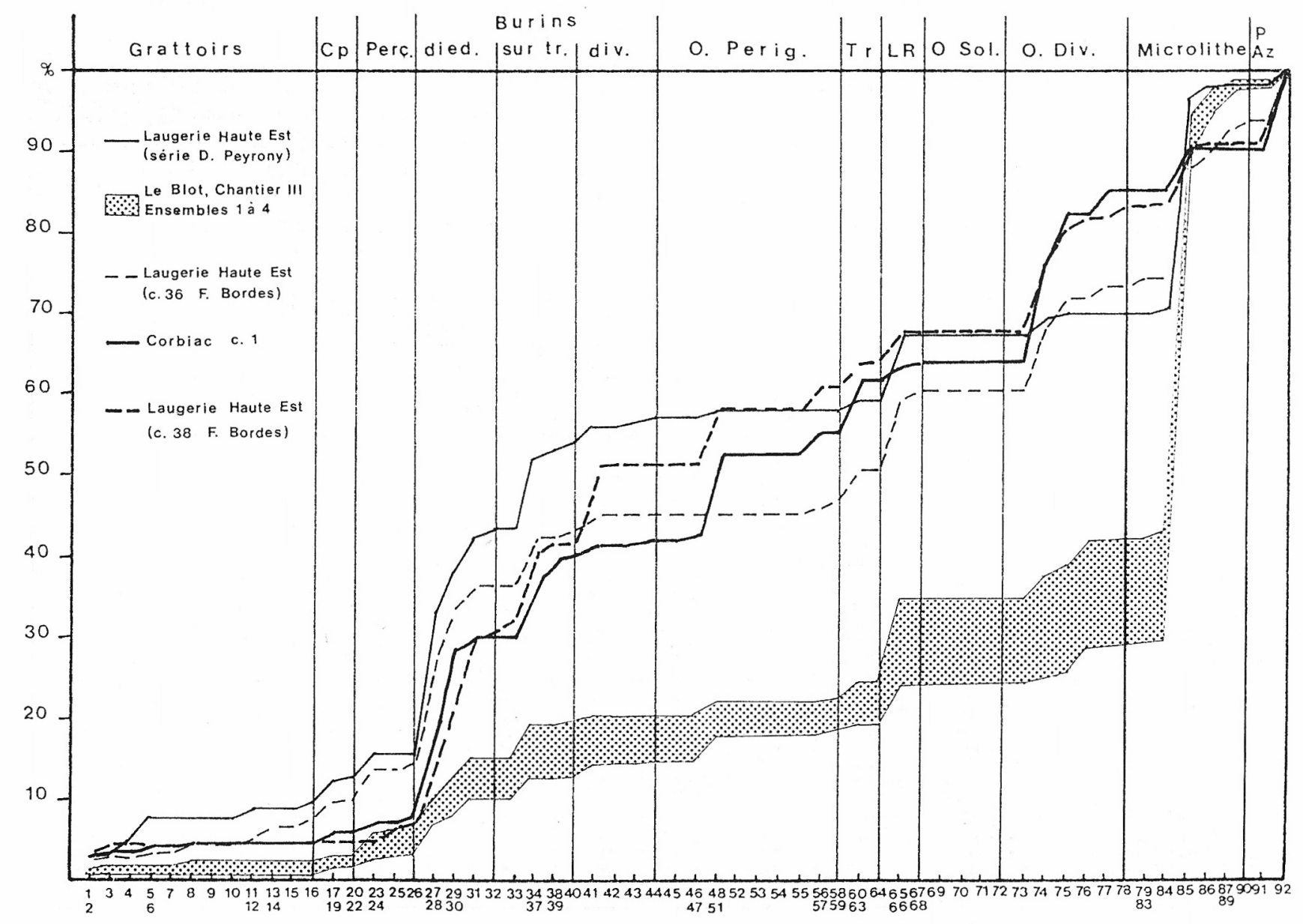

Fig. 2 - Diagrammes cumulatifs des industries du Blot, de Laugerie-Haute Est et de Corbiac (d'après F. Bordes et D. de Sonneville-Bordes, 1966, p. 120). 
$\left(n^{\circ} 76\right)$. En ce qui concerne les séries de LaugerieHaute Est, nous avons au préalable reclassé la nouvelle liste-type sur celle publiée en 1954 par D. de Sonneville-Bordes. Les indices typologiques obtenus sont rassemblés dans le tableau 5, puis traduits sous forme de graphiques cumulatifs sur la figure 3 . Les différences relevées sont sensibles mais pas fondamentales (si on exclut la couche 38 des fouilles F. Bordes pour sa faiblesse et la couche F des fouilles D. Peyrony au vu de son ancienneté). Notons tout d'abord des fréquences corrigées quasi équivalentes pour les perçoirs, les burins, les burins dièdres et les lames tronquées. Les grattoirs sont plus rares au Blot ; la fonction « gratter » étant cependant présente par les troncatures latéro-transversales des lames tronquées et de certains burins. Ces derniers apparaissent plus développés en Auvergne qu'en Périgord, tout comme les micro-

\begin{tabular}{|c|c|c|c|c|c|c|c|c|c|c|c|c|}
\hline SITE & IG & IP & IB & IBd & $\mathbf{I B t}$ & IPD & IT & $\mathrm{IRR}$ & 74.75 & $\mathbf{n}^{\circ} \mathbf{3 1}$ & $n^{\circ} 40$ & $n^{\circ} 41$ \\
\hline $\begin{array}{l}\text { Le Blot, c. } 1 \\
\text { Le Blot, c. } 2 \\
\text { Le Blot. c. } 3 \\
\text { Le Blot, c. } 4\end{array}$ & $\begin{array}{l}3.54 \\
1,66 \\
2,25 \\
2,50\end{array}$ & $\begin{array}{l}4.42 \\
4.42 \\
3.37 \\
5.00\end{array}$ & $\begin{array}{l}39,82 \\
45,30 \\
47.19 \\
44,17\end{array}$ & $\begin{array}{l}23.89 \\
29.28 \\
29.21 \\
27.50\end{array}$ & $\begin{array}{l}12,39 \\
11.05 \\
12,92 \\
11,67\end{array}$ & $\begin{array}{r}1,77 \\
3.31 \\
9,55 \\
10,83\end{array}$ & $\begin{array}{l}5,31 \\
4,97 \\
4.49 \\
5,83\end{array}$ & $\begin{array}{l}25,66 \\
26,52 \\
23.60 \\
18,33\end{array}$ & $\begin{array}{l}8,84 \\
8,29 \\
5,62 \\
6,67\end{array}$ & $\begin{array}{r}10,62 \\
5.52 \\
5.62 \\
7.50\end{array}$ & $\begin{array}{l}1.77 \\
2.21 \\
1.69 \\
3.33\end{array}$ & $\begin{array}{l}1.77 \\
4.42 \\
2.81 \\
2.50\end{array}$ \\
\hline Moyenne Le Blot & 2,49 & 4,30 & 44,12 & 27,47 & 12,01 & 6,37 & 5,15 & 23,53 & 7,36 & 7,32 & 2,25 & 2,88 \\
\hline $\begin{array}{l}\text { Laugerie-Haute Est, c. F } \\
\text { laugerie-Haute Est. c. } 38 \\
\text { laugerte-Haute Est, . } 36 \\
\text { Corbiac, c. } 1 \\
\text { Corbiac, c. } 1 \text { A }\end{array}$ & $\begin{array}{r}12,61 \\
2,86 \\
9,40 \\
7,34 \\
6,88\end{array}$ & $\begin{array}{l}4,75 \\
2,86 \\
3.45 \\
2,31 \\
1,60\end{array}$ & $\begin{array}{l}52,65 \\
42,86 \\
40,75 \\
44,02 \\
36,01\end{array}$ & $\begin{array}{l}35,10 \\
28.57 \\
29.47 \\
117.03 \\
22,48\end{array}$ & $\begin{array}{r}13,35 \\
4,29 \\
8.46 \\
111.20 \\
8,72\end{array}$ & $\begin{array}{r}3,30 \\
5,71 \\
12,36 \\
11,01\end{array}$ & $\begin{array}{l}3,10 \\
7,14 \\
7,52 \\
7.34 \\
9,40\end{array}$ & $\begin{aligned} 8,96 \\
5,71 \\
10,33 \\
6,56 \\
6,65\end{aligned}$ & $\begin{array}{r}3,11 \\
18,57 \\
12,23 \\
13.12 \\
14,68\end{array}$ & $\begin{array}{r}6,58 \\
12,86 \\
4.79 \\
0,39 \\
2,06\end{array}$ & $\begin{array}{l}2,01 \\
0.32 \\
0.77 \\
0.46\end{array}$ & $\begin{array}{l}3,11 \\
8,57 \\
2,24 \\
1,93 \\
1,60\end{array}$ \\
\hline
\end{tabular}

Tableau 5 - Indices typologiques « délamellisés » du Protomagdalénien et du Périgordien évolué de Corbiac.

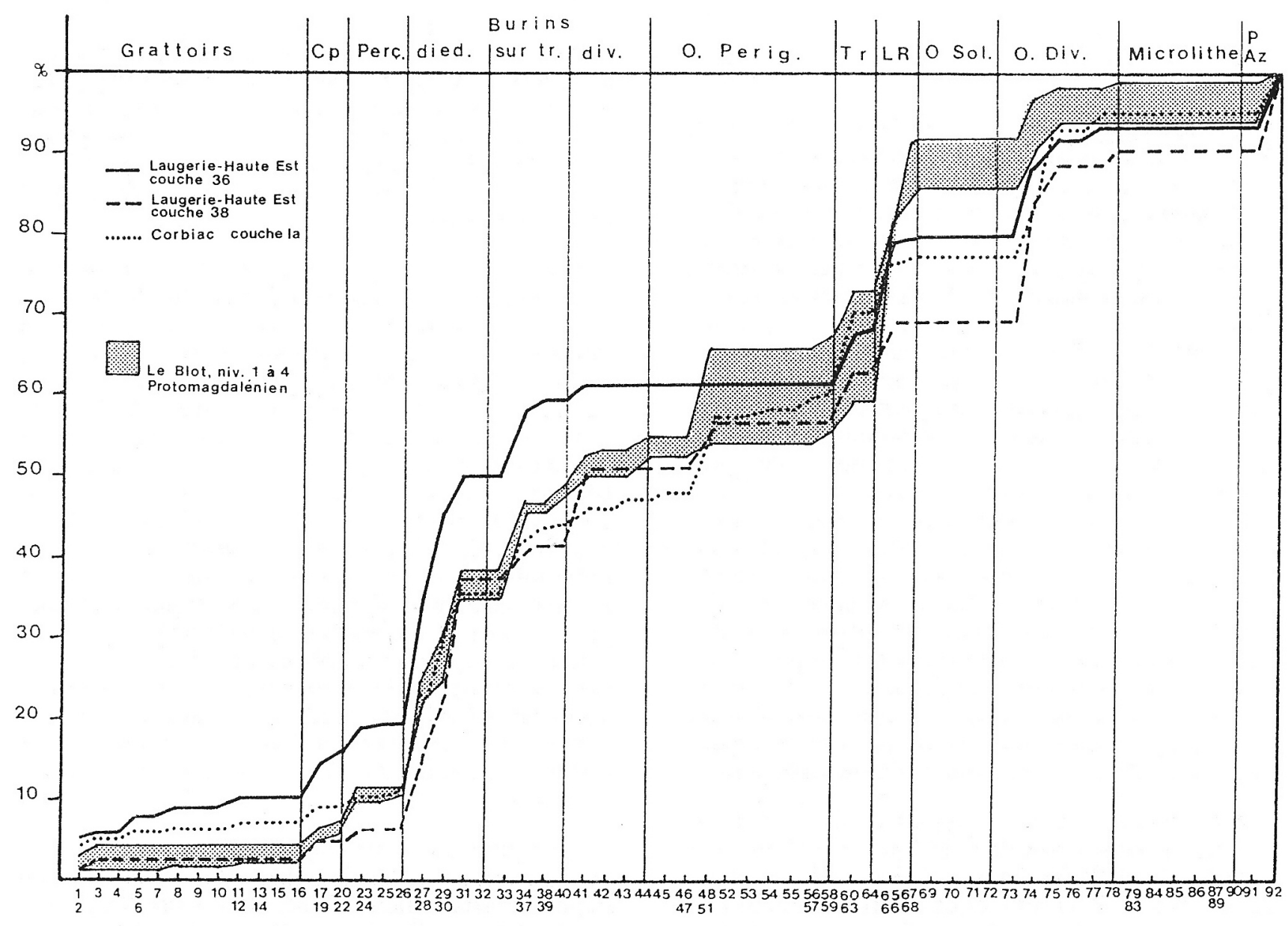

Fig. 3 - Diagrammes cumulatifs « délamellisés » du Blot, de Laugerie-Haute Est et de Corbiac. 
gravettes, absentes dans le niveau 36 de LaugerieHaute Est (vraisemblablement en corrélation avec le pourcentage élevé des microlithes en HauteLoire). Les encoches et denticulés possèdent un pourcentage relatif moins important au Blot qu'à Laugerie, peut-être est-ce là le signe d'une évolution. Enfin, il faut noter que la plus grande divergence provient des lames retouchées, très nombreuses en Haute-Loire, surtout sous leur forme bilatérale, parfois convergente. Toutefois, les modules de débitage du support des outils, la morphologie et le style général de l'industrie rapprochent clairement les deux sites. La retouche latérale, sur les lames comme sur les burins, la répartition entre les différentes catégories de burins, la présence de microgravettes au Blot, et à Laugerie (c. 38), constituent des éléments significatifs d'une parenté entre ces deux grands gisements situés à plus de $300 \mathrm{~km}$ l'un de l'autre mais attestent également d'une filiation du Protomagdalénien depuis un Gravettien très évolué comme celui de Corbiac par exemple.

\section{CONCLUSIONS}

L'étude typologique de l'industrie lithique du gisement du Blot confirme l'existence, pour la première fois, de la culture protomagdalénienne en stratigraphie. Les caractères généraux ont été définis à partir du décompte proposé par D. de Sonneville-Bordes : extrême rareté des grattoirs, développement moyen des burins, dont les exemplaires dièdres dépassent largement les types sur troncature retouchée, existence de nombreux burins multiples (dièdres et mixtes principalement), perduration des microgravettes, fréquence moyenne des lames tronquées et des encoches-denticulés, abondance des lames retouchées, surtout bilatérales, présence en quantité non négligeable de pièces esquillées, et enfin foisonnement des microlithes, à retouche croisée du dos. Des études complémentaires ont par ailleurs montré une évolution modérée dans la coupe (de bas en haut) : augmentation des burins, des lames tronquées et retouchées, des pièces esquillées et des outils « archaïques », au détriment des microlithes et des micropointes de la Gravette, l'ensemble étant parfois associé à un changement technologique (style de la retouche des lames retouchées et technique d'abattage du dos).

Par ailleurs, les séries lithiques du Blot apparaissent très proches de celles de Laugerie-Haute Est (couches 36 et 38 des fouilles F. Bordes et couche F des fouilles D. Peyrony), et de l'abri Pataud. Si l'on fait abstraction des quelques divergences concer- nant les grattoirs, les lames retouchées, les pièces esquillées et les microlithes lato-sensus (microgravettes et lamelles à dos), les diagrammes cumulatifs « délamellisés » confirment la similitude entre les niveaux Protomagdaléniens du Blot et de LaugerieHaute Est. L'originalité principale du Blot, par rapport à ses homologues du Périgord, se manifeste par l'abondance et l'hyper-microlithisme des lamelles à dos, la rareté des grattoirs, le développement un peu plus important des burins sur troncature retouchée, ou multiples, des microgravettes, et surtout des lames retouchées et des pièces esquillées.

Il est regrettable de ne pouvoir disposer, dans l'état actuel des connaissances, d'autres éléments de comparaisons en ce qui concerne le Protomag. dalénien, dans l'attente d'une publication exhaustive de l'abri Pataud, second site attribué avec certitude au Protomagdalénien, du Périgordien final de Corbiac et du Roc de Combe. Aux Tarterêts II, le débitage est trop lourd et trop épais. Le style des burins, de la retouche latérale des lames, plutôt écailleuse évoquerait plus vraisemblablement le Gravettien tel qu'il existe déjà en Ile-de-France (Schmider, 1971, 1983). Une industrie «évoluée » a été publiée par F. Champagne sur le site des Fieux à Miers. Dans l'immédiat, les burins sur troncature abondent, au contraire des burins dièdres, les microgravettes sont absentes et les lames retouchées sont assez rares. Là encore, il semble plutôt s'agir d'un Périgordien VI assez typique, malgré le pourcentage important des lamelles à dos, tronquées ou non (plus de $55 \%$ ).

Nous voudrions également aborder le problème de la répartition géographique du Protomag. dalénien. Il existe en effet deux sites bien repérés en Périgord, et un gisement dans le Massif Central, séparé des précédents par plus de $300 \mathrm{~km}$. La vallée de l'Allier semble, au cours du Pléistocène, l'unique voie de communication vers l'extérieur. Les hommes du Protomagdalénien se sont installés au Blot après avoir contourné l'ensemble du Massif, c'est-à-dire toute sa bordure occidentale. Ainsi peut-on justifier le décalage chronologique et climatique mis en évidence entre les deux provinces : le Protomagdalénien de Dordogne se situant dans une phase froide et sèche (Würm III-Périgord VIII), à caractère steppique tandis qu'au Blot, les occupations sont attribuées à une phase d'amélioration aboutissant à l'interstade de Laugerie (Moser, 1976). Cet écart peut expliquer partiellement une certaine variabilité dans l'outillage des hommes préhistoriques.

L'origine du Protomagdalénien du Blot reste encore sujette à caution. En effet, si la filiation depuis le Gravettien évolué, style Corbiac, ne fait aucun doute, celle-ci n'est pas décelée de manière 
péremptoire à partir des niveaux sous-jacents du Blot (couches 37 à 50), au contraire même puisque la coupure stratigraphique entre Périgordien et Protomagdalénien est très nette. L'hypothèse d'une industrie allochtone reste actuellement la plus plausible.

De la même manière, le devenir du Protomagdalénien est une question qui n'est pas élucidée. Au Blot, les derniers niveaux sont très évanescents et laissent place, après une zone stérile, à des industries radicalement différentes (Badegoulien). Certains auteurs ont développé une argumentation tendant à prouver l'origine du Magdalénien moyen et supérieur dans des faciès évolués de la fin du cycle gravettien (Rigaud, 1976). Comme nous le signalions précédemment (Bosselin et Djindjian, 1988 [1990]), les vestiges les plus tardifs du Protomagdalénien appartiennent à la plage 2200020000 BP tandis que les niveaux magdaléniens les plus anciens fournissent les dates de $17410 \pm 310 \mathrm{BP}$ au Roc de Marcamps, couche 4c (Ly-2292) et 17190 \pm 140 BP à Lascaux (GrN-1632). Le modèle de l'origine gravettienne du Magdalénien est réfutable sur la base d'un manque de données disponibles sur la période 20000-17500. Un élément de réponse se trouve peut-être dans le Pays Basque espagnol puisque, très récemment, A. Baldéon signale un gisement stratifié (La Cueva de Amalda) ayant livré au moins deux niveaux gravettiens : Périgordien Vc à Noailles $(27400 \pm 1000$ et $27400 \pm 1100$ BP [I11664 et I-11665]), Périgordien, vraisemblablement VI au vu de la typologie des burins $(19000 \pm 340$ et $17880 \pm 390$ BP [I-11663 et I-11372]), et Solutréen inférieur (à burins sur troncature, microgravettes, outils « archaïques », microlithes et seulement $1,54 \%$ d'outils solutréens $)(16090 \pm 240$ à $17580 \pm$ 440 BP [I-11435 et I-11355]) (Baldéon, 1990). C'est probablement dans cette région qu'il faudra chercher la filiation entre Gravettien et Magdalénien, confirmant ainsi la théorie de J.-Ph. Rigaud (1976).

\section{REMERCIEMENTS}

Je tiens à remercier ici toutes les personnes qui ont permis la réalisation de ce travail : en premier lieu, M. Henri Delporte pour la confiance qu'il m'a témoignée et les facilités qu'il m'a consenties lors de l'étude du matériel du Blot. J'associe également à cet hommage Mme D. de Sonneville-Bordes, qui m'a permis de consulter les séries F. Bordes issues du gisement de Laugerie-Haute Est, M. J.-J. CleyetMerle, Conservateur en Chef du Musée National de Préhistoire des Eyzies de Tayac, pour les séries D. Peyrony de ce même site. Enfin, je ne saurais oublier l'équipe du Musée de Saint-Germain-enLaye, en particulier D. Buisson pour m'avoir communiqué les résultats de son travail, encore inédit, sur le Périgordien du Blot.

Bruno Bosselin U.A. 880 du C.N.R.S. Musée des Antiquités Nationales Saint-Germain-en-Laye

Baldeon A. (1990) - Las Industrias de Los Niveles Paleoliticos. in "La Cueva de Amalda », Munibe, t. 42, 1990, pp. 64-115, 32 fig., 7 tabl., 12 photos.

BAZILE F. (1981) - Données récentes sur le Périgordien Supérieur en Languedoc. Études Quaternaires Languedociennes, Cahier n¹, 1981, pp. 19-30, 6 fig.

BORDES F. (1958) - Nouvelles fouilles à Laugerie-Haute Est : premiers résultats. L'Anthropologie, t. 62 (3-4), 1958, pp. 205 244, 27 fig.

Bordes F. (1968) - Emplacement de tentes du Périgordien supérieur évolué de Corbiac (près Bergerac), Dordogne. Quärtär, t. 19, 1968, pp. 251-262, 5 fig., 3 pl. h.-t.

Bordes F. (1978) - Le Protomagdalénien de Laugerie-Haute Est (fouilles F. Bordes). Bull. Soc. Préhist. Fr., t. 75 (11-12), 1978, pp. 501-522, 11 fig., 2 tabl.

Bordes F. et LABrot J. (1967) -- La stratigraphie du gisement du Roc de Combe (Lot) et ses implications. Bull. Soc. Préhist. Fr., t. 64 (1), 1967, pp. 15-28, 6 fig.

Bordes F. et Sonneville-Bordes D. de (1966) Protomagdalénien ou Périgordien VII ? L'Anthropologie, t. 70 (1-2), 1966, pp. 113-122, 5 fig.

BosseliN B. (1990) - La retouche «Protomagdalénienne » à la lueur des données récentes du site du Blot. Séminaire La pierre Préhistorique, Musée du Louvre, Laboratoire de Recherche des Musées de France, 13-14 décembre 1990, 54 p., 13 fig., 8 tabl., Prétirage.

Bosselin B. et DJindjIAN F. (1988) [1990] - Un essai de structuration du Magdalénien français à partir de l'outillage lithique. Bull. Soc. Préhist. Fr., t. 85, Études et Travaux (10-12), 1988 [1990], pp. 304-331, 11 fig.

Bouyssonie J. et Sonneville-Bordes D. de (1956) - L'abri $\mathrm{n}^{\circ} 2$ des Vachons. Gisement aurignacien et périgordien, commune de Voulgézac (Charente). Congrès Préhistorique de France, $\mathrm{XV}^{\mathrm{e}}$ session, Poitiers-Angoulème, 1956, pp. 271-309, 17 fig., 5 tabl.

BRICKER H.M. et DAVID N.C. (1984) - Excavation of the abri Pataud, Les Eyzies (Dordogne). The perigordian VI (level 3) assemblage. American School of Prehistoric Research, Peabody Museum, Harvard University, Bull. n 37, 1984.

Champagne F. et Jaubert J. (1979) - La grotte des Fieux à Miers (Lot). Bilan de 13 années de recherches. Congrès Préhistorique de France, $\mathrm{XXI}^{\circ}$ session, Montauban-Cahors, 1979 , t. 1, pp. 85-104, 13 fig., 1 tabl.

Clay R.B. (1968) - The Protomagdalenian culture. Unpublished Ph.D., Dissertation presented to the Dept. of Anthropology, Southern Illinois University, 1968, 2 vol., 660 p., 82 fig., 198 tabl.

Combier J. (1967) - Le Paléolithique de l'Ardèche dans son cadre paléoclimatique. Publication de l'Institut de Préhistoire de l'Université de Bordeaux, Mémoire 4, 1967, 462 p., 171 fig., 28 tabl., 5 cartes. 
Delporte H. (1966) - Le Paléolithique dans le Massif Central : I. Le Magdalénien des hautes vallées de la Loire et de l'Allier. Bull. Soc. Préhist. Fr., t. 63, Études et Travaux n 1, 1966 , pp. 181-207, fig.

Delporte H. (1969) - Protomagdalénien du Blot, commune de Cerzat (Haute-Loire). Congrès Préhistorique dé France, XIX ${ }^{\mathrm{c}}$ session, Auvergne, 1969, pp. 190-199, 7 fig.

DJINDJIAN F. (1980) - Construction de systèmes d'aide à la connaissance en archéologie préhistorique : structuration et affectation. Thèse de Doctorat d'État de l'Université de Paris I, 1980, 2 vol., 199 et 139 p., fig. et tabl.

FARrand W.R. (1975) - Analysis of the abri Pataud sediments. American School of Prehistoric Research, Peabody Museum, Harvard University, Bull. ${ }^{\circ}$ 30, pp. 27-68.

LAVILLE H. (1964) - Recherches sédimentologiques sur la paléoclimatologie du Würmien récent en Périgord. L'Anthropologie, t. 68 (1-2), 1964, pp. 1-48, 17 fig. et t. 68 (34), 1964, pp. 219-252, 25 fig.

Laville H. (1975) - Climatologie et chronologie $d u$ Paléolithique en Périgord: étude sédimentologique de dépôts en grottes et sous abris. Études Quaternaires, Publications de l'Université de Provence, Mémoire 4, 1975, 422 p., 181 fig., 6 tabl.

Le Tensorer J.-M. (1981) - Le Paléolithique en Agenais. Cahier du Quaternaire, $n^{\circ} 3$, Éditions du CNRS, Bordeaux, 1981, 526 p., 212 fig., 55 tabl.

MARGUERIE D. (1982) - Étude géologique du gisement préhistorique du Blot (Cerzat, Haute-Loire) : éléments de lithostratigraphie et palécologie. Diplôme d'Études Supérieures, École des Hautes Études en Sciences Sociales, Paris, 1982, 261 p., 78 fig., $6 \mathrm{pl}$.

Morala A. (1984) - Périgordien et Aurignacien en HautAgenais. Etude d'ensembles lithiques. Archives d'Écologie Préhistorique, École des Hautes Études en Sciences Sociales, Toulouse, Mémoire 7, 1984, 140 p., 42 fig., 9 photos

Morala A. (1987) - L'atelier Périgordien Supérieur de Rabier (Lanquais - Dordogne) : recherches sur l'origine géographique des occupants du site, sur les bases de la lithologie. Congrès International sur le silex, $\mathrm{V}^{\mathrm{c}}$ session, Bordeaux, oct.-nov. 1987, Prétirage, 21 p., 7 fig.

Moser F. (1976) - La sédimentologie des remplissages d'abris sous-basaltiques en Haute-Loire et ses implications climatiques au Würm récent et au Post-Würm. Diplôme E.P.H.E., Paris, $3^{\text {e }}$ section, 1976, 97 p., 45 fig., 1 tabl.

Moser F. (1976) - Les remplissages des grottes et abris sous basalte en Haute-Loire. in "La Préhistoire Française », Éditions du CNRS, 1976, t. I-1, pp. 271-274, 3 fig.

Movius H.L. Jr (1975) - Excavations at the abri Pataud, Les Eyzies (Dordogne). American School of Prehistoric Research, Peabody Museum, Harvard University, Bull. n 30, 1975 pp. 271-274.

Movius H.L. Jr et David N.C. (1970) - Burins avec modification tertiaire du biseau, burins-pointe et burins du Raysse à l'abri Pataud, Les Eyzies (Dordogne). Bull. Soc. Préhist. Fr. t. 67, Études et travaux n² 2, 1970, pp. 445-455, 6 fig.

Onoratini G. (1974) - Une lignée du Périgordien supérieur du Sud-Est de la France dans son cadre sédimento-climatique. Thèse de $3^{e}$ cycle de l'Université d'Aix-Marseille, 1974, 96 p., 18 fig., 30 pl., 4 pl. h.-t.

ONORATINI G. (1979) - Les industries de tradition gravettienne dans le Sud-Est de la France et en Ligurie. in " La fin des Temps Glaciaires en Europe », Colloque Talence, mai 1977 , Éditions du CNRS, 1979, t. 1, pp. 287-295, 4 fig., 4 tabl.
Onoratini G. (1982) - Préhistoire, Sédiments et Climats du Würm III à l'Holocène dans le Sud-Est de la France. Thèse de Docteur ès Sciences de l'Université d'Aix-Marseille III, 1982.

Peyrony D. (1952) - Correspondance : Laugerie-Haute Est Bull Soc. Préhist. Fr., t. 49, 1952, p. 567.

Peyrony D. et E. (1938) - Laugerie-Haute, près des Eyzies (Dordogne). Archives de l'Institut de Paléontologie Humaine, Mémoire 19, Masson Éditeur, Paris, 1938, 84 p., 56 fig., 7 pl.

Rigaud J.-Ph. (1976) - Données nouvelles sur le Périgordien Supérieur en Périgord. Études et Recherches Archéologiques de l'Université de Liège, $\mathrm{n}^{\circ}$ 13, Liège, t. 1, 1976, pp. 107-118, 2 fig.

Rigaud J.-Ph. (1982) - Le Paléolithique en Périgord: les données du Sud-Ouest Sarladais et leurs implications. Thèse de Doctorat d'État de l'Université de Bordeaux I, n 737, 1982, 2 vol., 494 p., 242 fig., 17 tabl.

SCHMider B. (1971) - Les industries lithiques du Paléolithique Supérieur en Ile-de-France. $6^{\mathrm{e}}$ suppl. à Gallia Préhistoire, Éditions du CNRS, Paris, 1971, 219 p., 109 fig., 9 tabl.

SCHMider B. (1983) - Le gisement magdalénien de la Pente des Brosses à Montigny-sur-Loing (Seine-et-Marne). Gallia Préhistoire, t. 26 (1), 1983, pp. 109-128, 14 fig., 3 tabl.

SONNEvilLe-Bordes D. de (1958-1959) — Problèmes généraux du Paléolithique Supérieur dans le Sud-Ouest de la France. L'Anthropologie, t. 62 (5-6), 1958, pp. 413-451, 20 fig. et t. 63 (1-2), 1959, pp. 1-36, 37 fig.

Sonneville-Bordes D. de (1960) -- Le Paléolithique Supérieur en Périgord. Imprimerie Delmas, Bordeaux, 1960, 2 vol., 558 p., 295 fig., 64 tabl., 10 cartes.

Sonneville-Bordes D. de et Perrot J. (1954-1956) - Lexique typologique du Paléolithique Supérieur. Bull. Soc. Préhist. Fr., t. 51 (7), 1954, pp. 327-335, 4 fig., t. 52 (2), 1955, pp. 76-79, 2 fig., t. 53 (8), 1956, pp. 408-412, 2 fig. et t. 53 (9), 1956, pp. 547-549, 5 fig.

Virmont J. (1981) - Le bassin de l'Allier au Paléolithique Supérieur. Industries et phases de peuplement. Thèse de $3^{\circ}$ cycle de l'Université d'Aix-Marseille, 1981, 378 p., 113 fig.

Liste des indices typologiques utilisés

IG Indice de grattoir.

IOc Indice d'outil composite.

IP Indice de perçoir.

IB Indice de burin.

IBd Indice de burin dièdre.

$31 \quad$ Indice de burin dièdre multiple.

IBt Indice de burin sur troncature retouchée.

$40 \quad$ Indice de burin multiple sur troncature retouchée.

$41 \quad$ Indice de burin multiple mixte.

BNo Indice de burin de Noailles.

IPD Indice de gravette + microgravette.

IT Indice de lame tronquée.

ILR Indice de lame retouchée.

ODv. Indice d'outils « archaïques » $\left(\mathrm{n}^{\text {os }} 74,75,77\right.$ et 78$)$

Enc. Indice d'encoche et denticulé.

Esq. Indice de pièce esquillée.

Ild Indice de lamelle à dos.

Mic. Indice de microlithes ( $\mathrm{n}^{\text {os }} 84$ à 89$)$

$\mathrm{O} / \mathrm{ld} \quad$ Indice d'outil sur lamelle (n ${ }^{\text {os }} 84$ et 86 à 89 ). 